\title{
UCRL-TR-202419
}

LAWRENCE LIVERMORE NATIONAL LABORATORY

\section{Computational Methods for Collisional Plasma Physics}

D. W. Hewett, A. B. Langdon, D. J. Larson, B.

F. Lasinski, C. H. Still

February 18, 2004 
This document was prepared as an account of work sponsored by an agency of the United States Government. Neither the United States Government nor the University of California nor any of their employees, makes any warranty, express or implied, or assumes any legal liability or responsibility for the accuracy, completeness, or usefulness of any information, apparatus, product, or process disclosed, or represents that its use would not infringe privately owned rights. Reference herein to any specific commercial product, process, or service by trade name, trademark, manufacturer, or otherwise, does not necessarily constitute or imply its endorsement, recommendation, or favoring by the United States Government or the University of California. The views and opinions of authors expressed herein do not necessarily state or reflect those of the United States Government or the University of California, and shall not be used for advertising or product endorsement purposes.

This work was performed under the auspices of the U.S. Department of Energy by University of California, Lawrence Livermore National Laboratory under Contract W7405-Eng-48. 


\title{
Computational Methods for Collisional Plasma Physics
}

\author{
Dennis W. Hewett, A. B. Langdon, D. J. Larson, B. F. Lasinski, C. H. Still
}

Modeling the high density, high temperature plasmas produced by intense laser or particle beams requires accurate simulation of a large range of plasma collisionality. Current simulation algorithms accurately and efficiently model collisionless and collision-dominated plasmas. The important parameter regime between these extremes, semi-collisional plasmas, has been inadequately addressed to date. LLNL efforts to understand and harness high energy-density physics phenomena for stockpile stewardship require accurate simulation of such plasmas. We have made significant progress towards our goal: building a new modeling capability to accurately simulate the full range of collisional plasma physics phenomena.

Our project has developed a computer model using a two-pronged approach that involves a new adaptive-resolution, "smart" particle-in-cell algorithm: complex particle kinetics $(\mathrm{CPK})$; and developing a robust 3D massively parallel plasma production code $\mathrm{Z} 3$ with collisional extensions. Our new CPK algorithms expand the function of point particles in traditional plasma PIC models by including finite size and internal dynamics. This project has enhanced LLNL's competency in computational plasma physics and contributed to LLNL's expertise and forefront position in plasma modeling. The computational models developed will be applied to plasma problems of interest to LLNL's stockpile stewardship mission. Such problems include semi-collisional behavior in hohlraums, high-energydensity physics experiments, and the physics of high altitude nuclear explosions (HANE).

Over the course of this LDRD project, the world's largest fully electromagnetic PIC calculation was run, enabled by the adaptation of Z3 to the Advanced Simulation and Computing (ASCI) White system. This milestone calculation simulated an entire laser illumination speckle, brought new realism to laser-plasma interaction simulations, and was directly applicable to laser target physics. For the first time, magnetic fields driven by Raman scatter have been observed. Also, Raman rescatter was observed in 2D. This code and its increased suite of dedicated diagnostics are now playing a key role in studies of short-pulse, high-intensity laser matter interactions. In addition, a momentumconserving electron collision algorithm was incorporated into Z3. Finally, Z3's portability across diverse MPP platforms enabled it to serve the LLNL computing community as a tool for effectively utilizing new machines.

\section{Complex particle kinetics}

Complex particle kinetics (CPK) has demonstrated the ability to accurately simulate partially collisional plasmas in multiple dimensions. The particles evolve selfconsistently in either the fluid or fully collisionless limit. They are then spit in velocity and/or position space, producing more particles. This procedure effectively probes phase space in order to capture emerging features of the simulation. We then employ aggressive merging in order constrain the number of particles. The merging procedure retains the first and second moments of the distribution function. The basic algorithms 
governing particle fragmentation, merging, and internal dynamics are described in UCRL-JC-148321 [1].

Partially-collisional physics is modeled using a new Coulomb collision algorithm, appropriate for particles with an internal velocity distribution, described in UCRL-JC148993 [2]. This algorithm reduces the required number of particle pairings per time step while reproducing known partially collisional, counter-streaming ion beam behavior. We have recently extended this algorithm to model more than two species $(n>2)$.

We have compared results from a CPK simulation with those of the well-known hydrodynamics code Cale for a laser-ionized carbon foam striking a wall. The simulation parameters are similar to parameters used in a series of experiments recently performed by LLNL personal. A slab of carbon foam with a density of $0.44 \mathrm{~g} / \mathrm{cc}$ is ionized by a laser and set into motion at $2 \times 10^{6} \mathrm{~cm} / \mathrm{s}$. The slab expands due to internal pressure and the presence of an electric field due to electron/ion charge separation. The slab runs into a barrier at the end of the simulation. In the experiment the carbon slab impacts a piece of aluminum. The pressure history on the impact side of the aluminum is inferred from a time history of the aluminum plate velocity. The experimental data typically shows the pressure rising earlier in time and peaking at a lower value than the hydro-code simulation predicts. Figure 1 below shows three snapshots of the carbon density. The density evolution demonstrates the effect of material reaching the far end $(x=0.05 \mathrm{~cm})$ of the simulation earlier than Cale predicts. The fluid code assumes full collisionality, while the CPK simulation spans a fully to partially collisional parameter space.
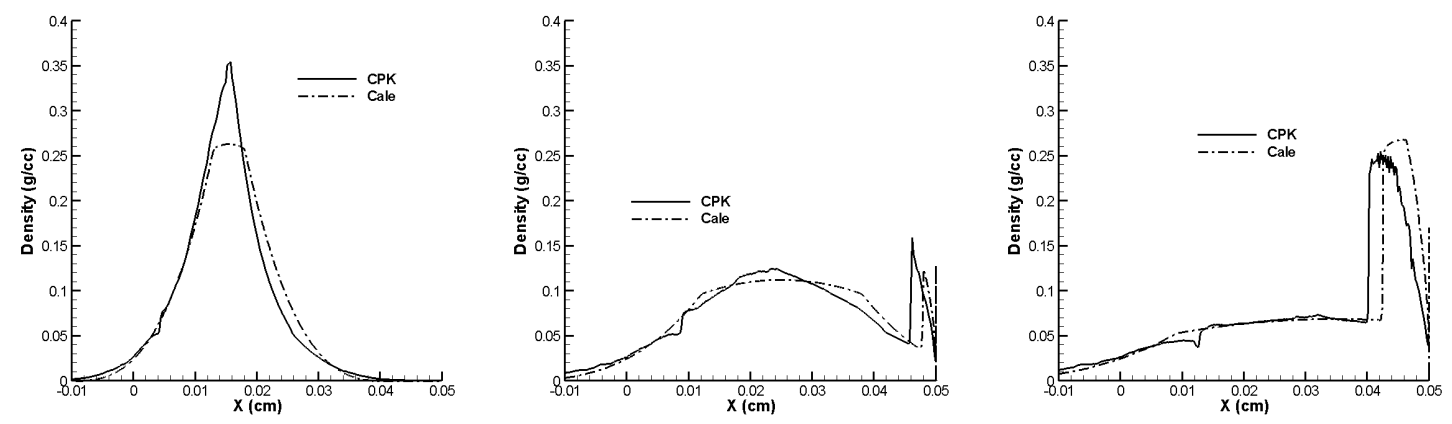

Figure 1. Density vs position at 5,10, and $15 \mathrm{~ns}$ from CPK and Cale simulations.

The pressure history from this simulation is plotted in Figure 2 (a). The earlier and softer rise and the reduced peak pressure seen in the CPK simulation is consistent with the general trend seen in experimental results. Figure 2 (b) shows the pressure history obtained via Cale simulation and experiment for a $0.1 \mathrm{~g} / \mathrm{cc}$ carbon foam. 


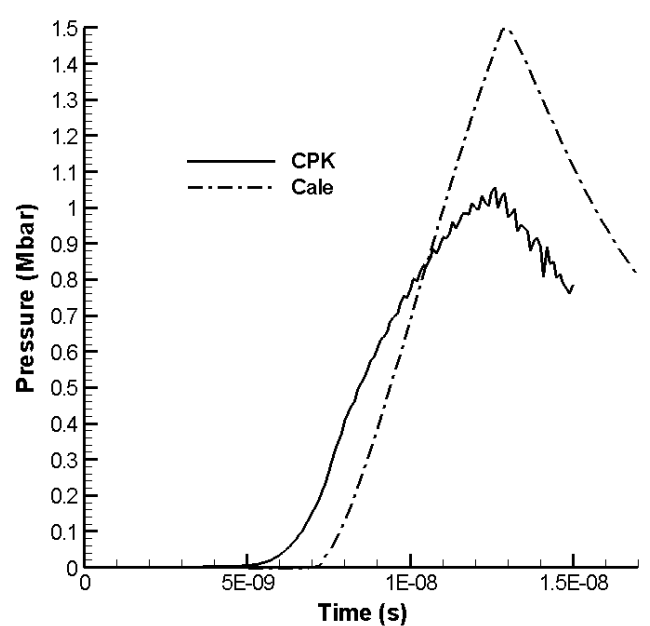

(a)

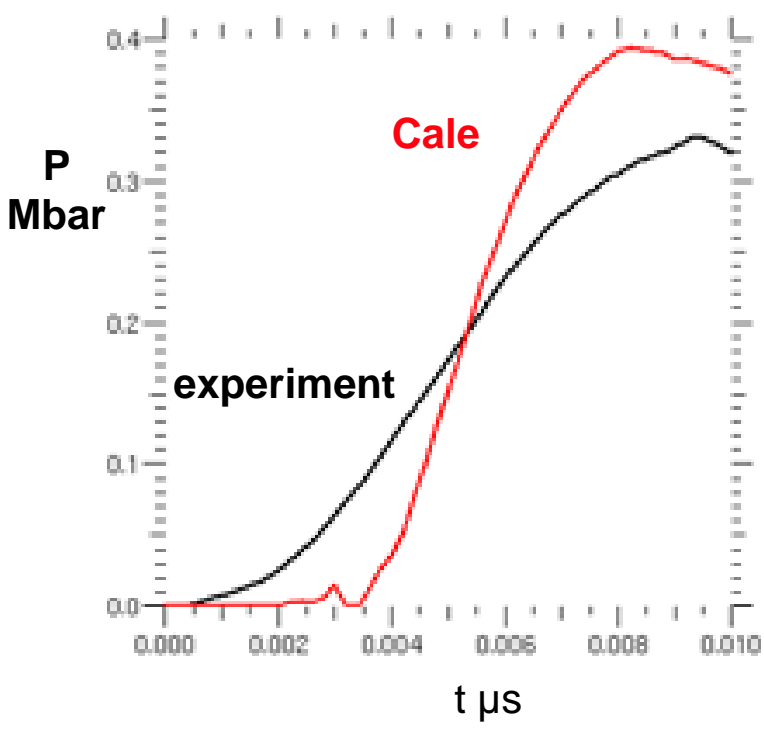

(b)

Figure 2: (a) Pressure history for simulation shown in Figure 1. (b) Pressure history for experiment 27027 showing a Cale calculation using EOS78 and the experimental data (Courtesy of John Edwards, LLNL.)

The first two-dimensional simulation we performed using the CPK algorithm involves the collision and subsequent interaction of counter-streaming plasmas. The parameters are taken from an experiment and simulations reported by Wan, et. al. [3]. The geometry is shown in Figure 3 (a). A laser beam strikes two gold slabs arranged at 90 degrees with a fixed gap between them. The simulation used a gap of $250 \mu \mathrm{m}$. The plasma blows off and collides at the symmetry plane late in time. For a 50-times ionized gold plasma with $T_{e}=3 \mathrm{keV}$ and $n_{i}=2 \times 10^{19} \mathrm{~cm}^{-3}$, our simulation results are consistent with those reported in [3], showing an off-axis electron density peak of $\sim 7 \times 10^{20} \mathrm{~cm}^{-3}$ at $1 \mathrm{~ns}$. A plot of the density at $1 \mathrm{~ns}$ is shown in Figure 3 below along with a diagram of the simulation geometry. 


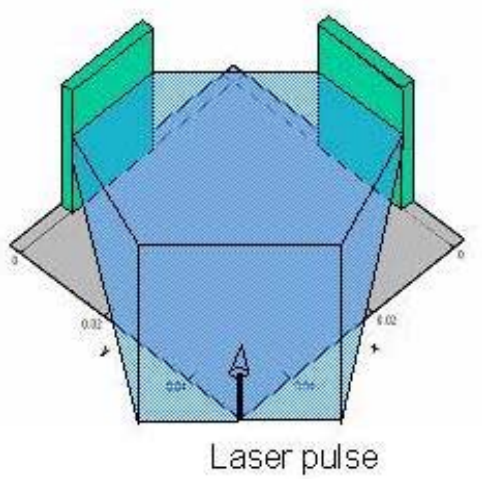

(a)

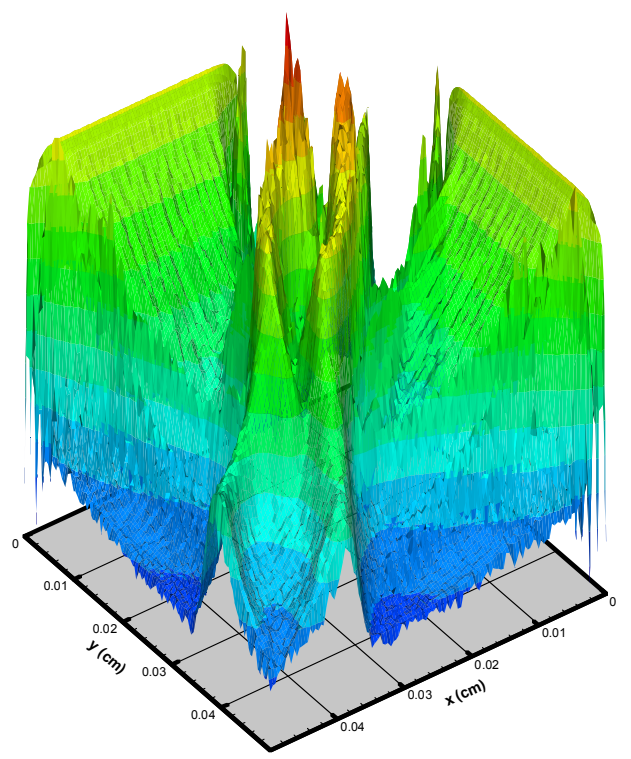

(b)

Figure 3: (a) Simulation geometry. (b) Density at 1 ns showing off-axis peaks.

\section{$\mathbf{Z 3}$}

Z3 has become a mature 3D massively parallel PIC code that runs on many platforms. Its increased set of dedicated diagnostics allows us to explore new physics in both the longpulse and short-pulse regimes of laser plasma interactions.

We have demonstrated that simulating an entire $\mathrm{f} / 8$ speckle is now feasible. With $\mathrm{Z} 3$ on 512 processors we have modeled an $\mathrm{f} / 4$ speckle as a capability demonstration; that simulation has a simulation volume of $25 \lambda_{0} \times 25 \lambda_{0} \times 153 \lambda_{0}$ and $7.6 \times 10^{9}$ particles were followed. This achievement shows that direct comparison of PIC simulations with single-speckle experiments and more macroscopic simulations relevant to the long-pulse regime is now feasible.

Recent results in the long pulse parameter regime are described in Reference [4]. In Fig. 4 , we show the two components of $\mathrm{B}$ (theta) from a $3 \mathrm{D}$ simulation for parameters of a high-intensity small hohlraum. This magnetic field is consistent with a current of forward going electrons, as is produced by both back and forward Stimulated Raman Scatter (SRS). We needed to implement time filtering and then employ further filtering to isolate the component in the same plane as the linearly polarized laser light. These magnetic fields are strong enough to confine the heated electrons within the speckle and thus affect subsequent evolution of SRS.

In a large $2 \mathrm{D}$ simulation for the same parameters, we again isolated this static magnetic field. We ran this simulation for 2 ps and showed that there is significant SRS before the laser traverses the entire speckle. There are bursts in time of this instability, as shown in Fig. 5. We identify the signal that begins past1 ps as the rescatter of Raman forward 
scatter. This process has been observed in 1D simulations, as described in Reference [5], but this is the first observation in $2 \mathrm{D}$.

We are also using Z3 to model the production of hot electrons and energetic protons in short-pulse laser plasma interactions in $2 \mathrm{D}$ and 3D simulations. We are currently modeling obliquely incident light on relativistically overdense plasma with varying amounts of underdense plasma (as generated by laser pre-pulses) in the beam path. Figure 6 shows the hot electron phase space and the $2 \omega_{0}$ pulses consistent with the $J \times B$ mechanism.

These results demonstrate the evolution of Z3 into a mature MPP code which runs on many computer platforms and the importance of a suite of dedicated diagnostics, both from the running code and as a result of post-processing (often in a parallel environment), in obtaining significant new scientific results.

\section{Summary}

We have developed a novel simulation capability using a two-pronged approach. Z3 has evolved into a mature MPP code with a suite of dedicated diagnostics and is enabling the discovery of new plasma physics phenomena. The complex particle kinetics (CPK) algorithm has reproduced known results spanning a large range of collisionality. We will apply this method to emerging problems of interest in high energy density physics, stockpile stewardship, and advanced concepts.

\section{References}

[1] D. W. Hewett, "Fragmentation, Merging, and Internal Dynamics for PIC Simulation with Finite Size Particles", J. Comp. Phys. 189 (2003) 390-426.

[2] D. J. Larson, “A Coulomb collision model for PIC plasma simulation”, J. Comp. Phys. 188 (2003) 123-138.

[3] A.S.Wan, et.al., "Electron density measurement of a colliding plasma using soft x-ray laser interferometry", Phys. Rev. E. 55 (5) (1997) 6293-6296.[4] B. F. Lasinski, C. H. Still, A. B. Langdon, D. E. Hinkel, and E. A. Williams, "Raman generated magnetic fields in laser light speckles", in Inertial Fusion Sciences and Applications 2003, Elsevier 2004.

[5] A. B. Langdon and D. E. Hinkel, "Nonlinear evolution of stimulated scatter in hightemperature plasmas", Phys. Rev. Lett. 89, 015003 (2002). 


\section{Figures}

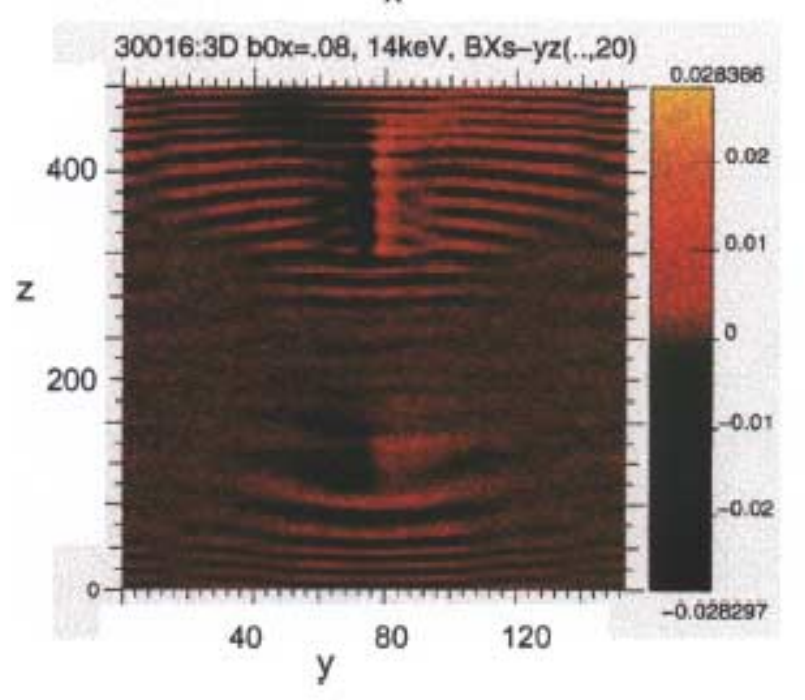

Figure 4: two components of $\mathrm{B}$ (theta) from a 3D simulation for parameters of a highintensity small hohlraum.

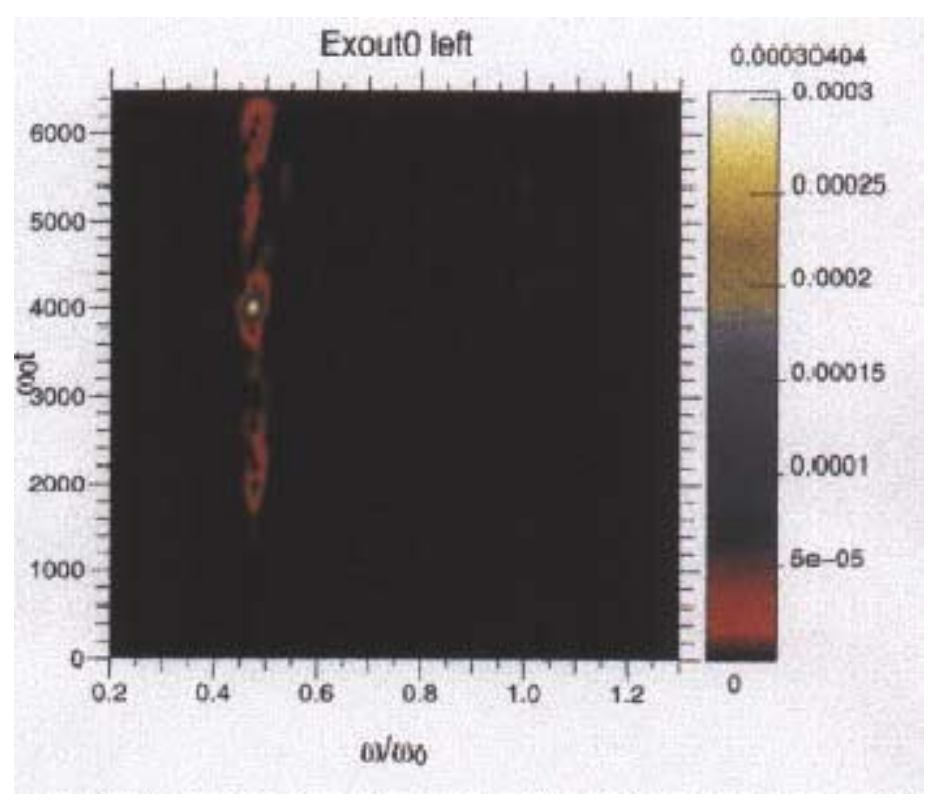

Figure 5: Plots of the frequency (horizontal axis) vs time (vertical axis) of the emitted light at the input plane for a $2 \mathrm{D}$ simulation at $0.2 n_{c}, 14 \mathrm{keV}$, and intensity $7 \times 10^{16} \mathrm{~W} / \mathrm{cm}^{2}$. 


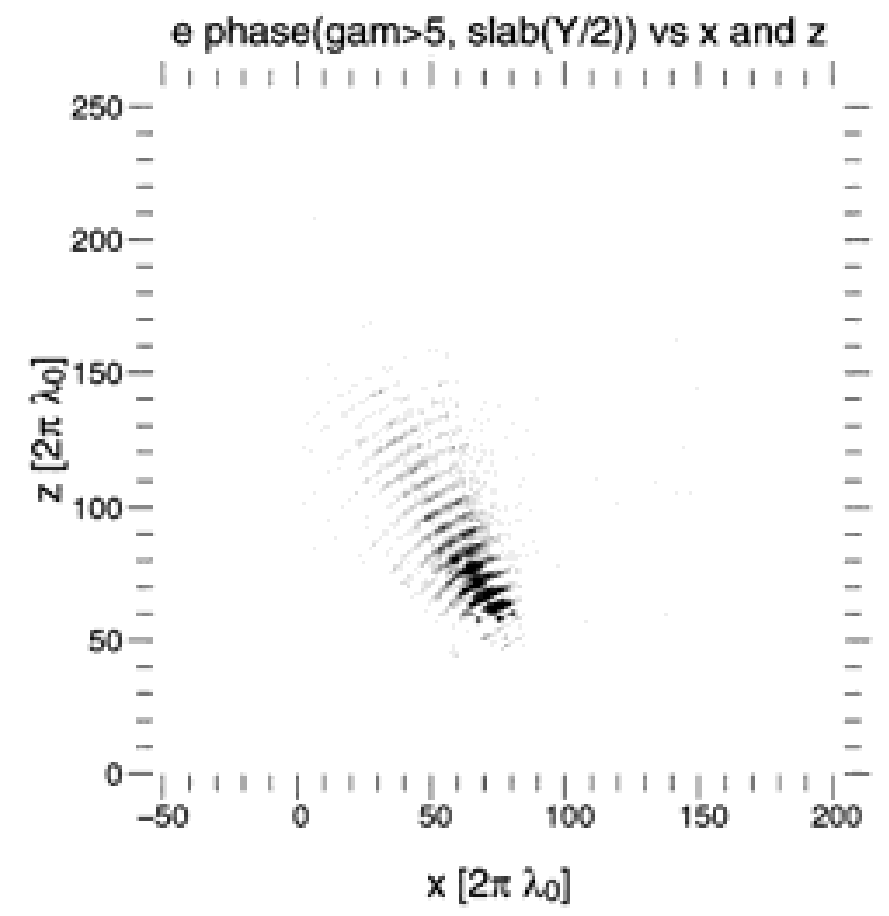

Figure 6: Hot electron phase space and the $2 \omega_{0}$ pulses consistent with the $J \times B$ mechanism. 